\title{
The effect of noise exposure on the aging ear
}

\author{
Geoffrey Shone, Richard A. Altschuler, Josef M. Miller and Alfred L. Nuttall \\ Kresge Hearing Research Institute, The University of Michigan, Ann Arbor, Michigan, U.S.A.
}

(Received 27 September 1990; accepted 12 June 1991)

\begin{abstract}
The effect of noise exposure on auditory sensitivity and inner ear morphology was compared in aged and young mature mice. Hearing thresholds were obtained by auditory evoked brain stem responses (ABR) before and after noise exposure, and hair cell loss was quantified. The study was done in two parts: first to assess the effect of noise exposure on subjects with preshycusis, and second to assess its effect on aged subjects without measurable presbycusis. In the first experiment C57BL/6 mice, with an age-related hearing loss, were used as a model for presbycusis. C57BL/6 mice exhibiting presbycusis were more susceptible to noise injury than age-matched $\mathrm{CBA} / \mathrm{Ca}$ mice. In the second experiment $\mathrm{CBA} / \mathrm{Ca}$ mice were used. These mice retain normal hearing even with advancing age. The aged $\mathrm{CBA} / \mathrm{Ca}$ mice had the same susceptibility to noise injury as young $\mathrm{CBA} / \mathrm{Ca}$ mice.
\end{abstract}

Mice; Noise-induced hearing loss; Aging; Presbycusis

\section{Introduction}

The interaction of presbycusis and noise-induced cochlear injury is an important question from many points of view, including occupational health. It determines the disability (and the level of compensation) of aged employees injured by noise, and may affect the need for aged people to take extra precautions against noise injury. It is also of relevance in demographic and other studies of noise-induced hearing loss, where a factor for presbycusis has to be used when quantifying noise injury.

To date, there has been little research on this issue. Human studies provide conflicting results; several studies suggest there is an additive effect of noise injury and presbycusis (McRae, 1971; Welleschik and Raber, 1978 ), and others suggest that the aged ear with presbycusis is less susceptible to noise injury than the normal ear (Novotny, 1975a,b; Lutovats, 1969). Schmiedt et al. (1990) have shown rearing gerbils in noise leads to degraded two-tone suppression responses in auditory nerve fibers. Henry (1982a,b; 1983b) has studied the effect of old age alone (without presbycusis) on susceptibility to noise injury in mice and found that aged mice were slightly less susceptible than young adult mice.

Investigation into the interaction of aging and noise exposure requires an animal model which ideally would

Correspondence to: Josef M. Miller, Kresge Hearing Research Institute, The University of Michigan, 1301 E. Ann Street, Ann Arbor, MI 48109-0506, U.S.A. Fax: (313) 764-0014. mirror the changes seen in aging and in noise injury in the human. Several animals have been studied to assess their suitability as a model for human age-related hearing loss. Although Mills et al. (1990) have found an age dependent change in cochlear sensitivity in the gerbil, most of the data in the literature have been obtained from mice, some strains of which have age-related physiological and anatomical cochlear changes similar to those seen in humans, including progessive basal to apical hair cell loss (Mikaelian, 1979; Henry, 1982c; Shnerson and Pujol, 1981; Chole and Henry, 1983; Church and Shucard, 1986; Henry and Lepkowski, 1978; Hunter and Willott, 1987; Henry, 1983b). Even the non-human primates do not mimic human presbycusis. They show less hearing loss (Bennett et al., 1983) and a different pattern of hair cell loss (Hawkins et al., 1985). Both guinea-pigs (Dum et al., 1980a,b) and rats (Crowley et al., 1982) exhibit only very minor presbycusis at the end of their lives, and in both cases the pattern of the hair cell loss is different from that in the human, progressing from the apex toward the base.

In the current study, $\mathrm{CBA} / \mathrm{Ca}$ and $\mathrm{C} 57 \mathrm{BL} / 6$ mice were used to examine the interaction of aging and noise injury in two phases: first, to assess the effect of noise exposure in combination with presbycusis, and second, to assess its effect on aged mice without presbycusis, i.e., still with normal-hearing. CBA/Ca mice maintain hearing sensitivity and hair cell populations throughout their lifespan, viz., for about two years (Henry and Chole, 1980; Wenngren and Anniko, 1988; Henry, 1982c; Willott, 1986; McGinn et al., submitted). C57BL/6 mice have been found to be an appropriate 
model for human presbycusis. They develop gradual hearing loss during young adulthood. Hearing loss begins at two months of agc, rcaches moderate levels by six months, and becomes profound by 15 months (Henry and Chole, 1980; Henry, 1982b; Shnerson and Pujol, 1981; Willott, 1986). The anatomical pattern of the hair cell loss is comparable with that seen in human presbycusis.

\section{Materials and Methods}

\section{Subjects and protocol}

Forty male CBA/Ca (aged 6 and 21 months) and C57BL $/ 6$ (aged 6 months) mice obtained from Charles River laboratories, were used in this investigation. All experimental protocols were reviewed and approved by the University of Michigan Committee on Use and Care of Animals, and the subjects were maintained throughout the study in AAALAC approved facilities.

Study 1 . The effect of noise exposure on six-monthold CBA / Ca mice (normal-hearing) was compared with the effect on six-month-old C57BL/6 mice. At this age, the latter have a significant degree of age-related hearing loss, modelling human presbycusis.

Study II. The effect of noise exposure on six-monthold CBA/Ca mice was compared with the effect on normal-hearing 21-month-old mice of the same strain, so that the effect of old age alone, without presbycusis. could be assessed. Each group of mice comprised ten subjects; half were noise-exposed and half were unexposed age- and strain-matched controls. All subjects underwent electrophysiological assessment to determine their baseline sensitivity. Discrete frequency ABR 'audiograms' were constructed. The pre-noise thresholds of the noise-exposed mice were not significantly different from the thresholds of the non-noise-exposed controls. Noise-exposed mice had post-noise thresholds measured at one hour and at one week after noise exposure. Both the noise-exposed and the control mice were then sacrificed to determine the distribution of hair cell loss (cytocochleograms).

\section{Noise exposure}

The mice were exposed individually in a well ventilated calibrated soundexposure facility. Broadband $(500-40,000 \mathrm{~Hz})$ noise was presented for $45 \mathrm{~min}$ at 101 dB SPL, measured with a sound level meter having flat frequency weighting. Exposures were performed with awake subjects, $24 \mathrm{~h}$ following pre-exposure electrophysiological assessment. The typical sound level across frequencies from a single location within the exposure cage is shown in Fig. 1. The sound spectrum levels, determined with a wave analyzer, were similar at each location measured.

\section{Electrophysiological measures}

All mice had their thresholds determined on two separate occasions, and the results of the two measurements averaged to obtain their pre-exposure baseline thresholds. Post-noise thresholds were measured one hour following exposure and again after one week, to obtain measures of both temporary and permanent noise-induced threshold shifts (TTS and PTS).

For ABR measurements, the mice were anesthetized with ketamine $80 \mathrm{mg} / \mathrm{kg}$ and xylazine 14 $\mathrm{mg} / \mathrm{kg}$. Measurements took place in an acoustically and electrically shielded booth (IAC), with the subjects lying prone and protected from heat loss by wrapping the body in a small blanket. Acoustic stimuli were delivered via a modified aural speculum loosely fitted just inside the ear canal of the left ear without distorting the meatus. The speculum was fitted with a calibrated probe tube on a $1 / 2$ inch $B$ and $K$ microphone for measurement of the sound level of the stimuli within the meatus.

Sound stimuli consisted of tone bursts of alternating polarity with a trapezoidal envelope, linear rise and fall times of $1 \mathrm{~ms}, 15 \mathrm{~ms}$ overall duration, and presented at a rate of $10 / \mathrm{s}$. The frequencies tested were $8,12,16$, 20,24 , and $32 \mathrm{kHz}$.

Electrophysiological responses were recorded from subcutaneous needle electrodes placed at the vertex and at the retro-auricular region, with the ground placed behind the opposite ear. Physiological signals were amplified $(10,000 \times)$, filtered $(0.3-3.0 \mathrm{~K}$ bandwidth) using a Grass P15 ampifier and each measurement was based on an average of 1024 sweeps ( $\mathrm{MI}^{2}$ signal processor). Each series of stimuli were begun at supra-threshold level and the attenuation increased in $10 \mathrm{~dB}$ steps; threshold was defined as $5 \mathrm{~dB}$ above the level of the last clearly visible, reproducible response.

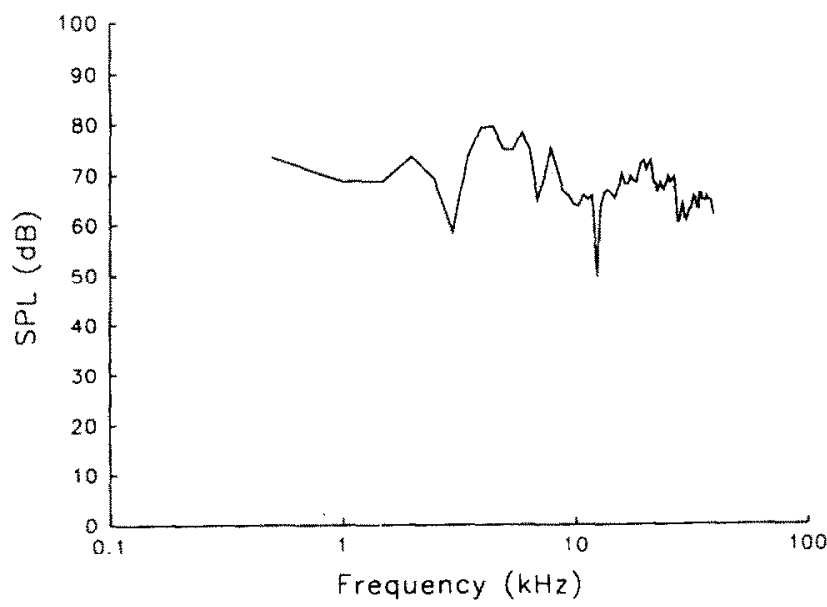

Fig. 1. Typical sound spectrum recorded from a single site in the exposure chamber. 


\section{Histological measures}

For assessment of hair cell losses, mice were heavily anesthetized with pentobarbital and then perfused systemically with $4 \%$ paraformaldehyde in phosphate buffer. After rapid removal of the temporal bones, the isolated cochleas were perfused with the same fixative via the oval and round windows, under an operating microscope. The cochleae were then stained with $1 \%$ osmium and dehydrated in a series of alcohols and embedded in resin. Excess resin was drilled away and serial half-turns of the cochlea dissected. After further drilling to thin each half-turn, the specimens were mounted and hair cells counted on a Leitz Dialux photomicroscope using a $40 \times$ or $25 \times$ objective and differential interference contrast optics.

\section{Results}

\section{Auditory thresholds}

Fig. 2 illustrates the ABR thresholds of the 6- and 21 -month-old CBA/Ca mice prior to and at one hour following noise exposure (TTS). Each data point represents the mean of five subjects. The older CBA/Ca animals demonstrated higher pre-exposure thresholds (approximately $10 \mathrm{~dB}$ ) at the two highest frequencies tested. No difference in noise-induced threshold shift was observed between the young and old exposure groups. Both groups demonstrated a statistically significant threshold shift of 30 to $35 \mathrm{~dB}$ for all frequencies tested above $12 \mathrm{kHz}$. Fig. 3 shows the pre- and post-exposure functions one week following the noise exposure. No PTS was induced by the noise exposure in either group. Moreover, the original decreased sensitivity of the older group observed pre-exposure was closely replicated.

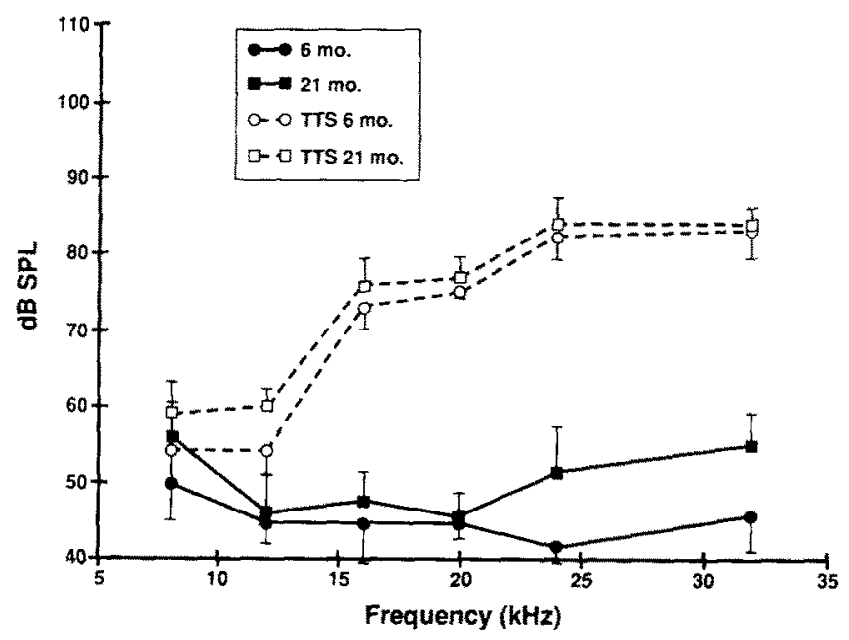

Fig. 2. Temporary threshold shift (one hour after noise) of 6-monthold mice, (CBA/Ca) compared with 21 -month-old mice $(\mathrm{CBA} / \mathrm{Ca})$.

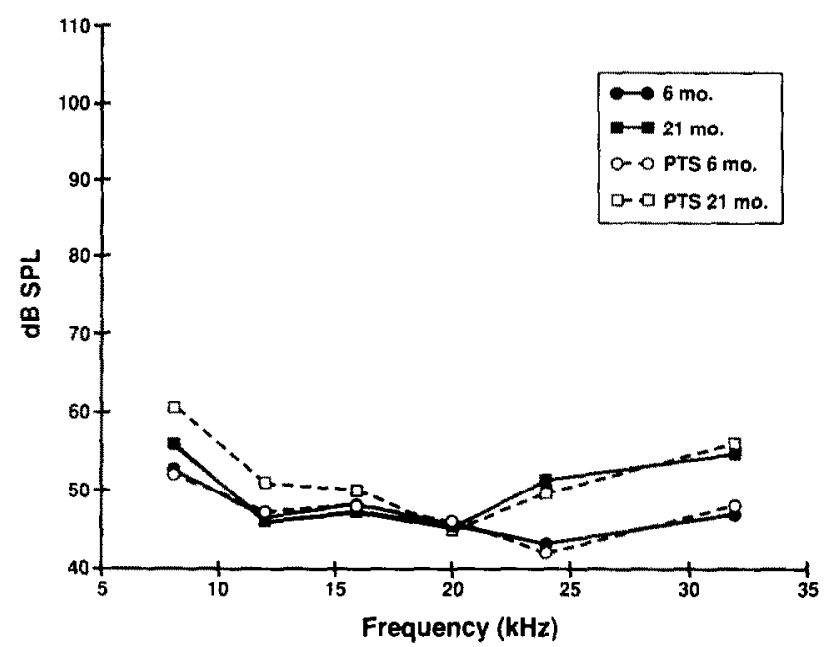

Fig. 3. Permanent threshold shift (one week after noise) of 6-monthold and 21-month-old CBA/Ca mice.

Fig. 4 illustrates the ABR threshold sensitivity functions for six month $\mathrm{CBA} / \mathrm{Ca}$ (normal) and six month C57BL/6 (with presbycusis) subjects prior to and one hour following the noise exposure. For all test frequencies of $12 \mathrm{kHz}$ and above, the C57BL/6 mice showed higher pre-noise thrcsholds than the $\mathrm{CBA} / \mathrm{Ca}$ group. This decreased sensitivity in C57BL/6 mice increased across frequency from $13 \mathrm{~dB}$ at $12 \mathrm{kHz}$ to more than 35 $\mathrm{dB}$ at frequencies above $20 \mathrm{kHz}$. At $32 \mathrm{kHz}$, no measureable response was obtained, to the limits of our equipment $(105 \mathrm{~dB} @ 32 \mathrm{kHz})$ in the $\mathrm{C} 57 \mathrm{BL} / 6$ group.

One hour following noise exposure both groups exhibited a clear TTS. After noise exposure, the C57BL $/ 6$ subjects exhibited a consistent 10-15 dB higher threshold than the $\mathrm{CBA} / \mathrm{Ca}$ subjects across frequency. However, the observed TTS was smaller in these subjects than that seen in $\mathrm{CBA} / \mathrm{Ca}$ subjects for high frequencies (above $16 \mathrm{kHz}$ ).

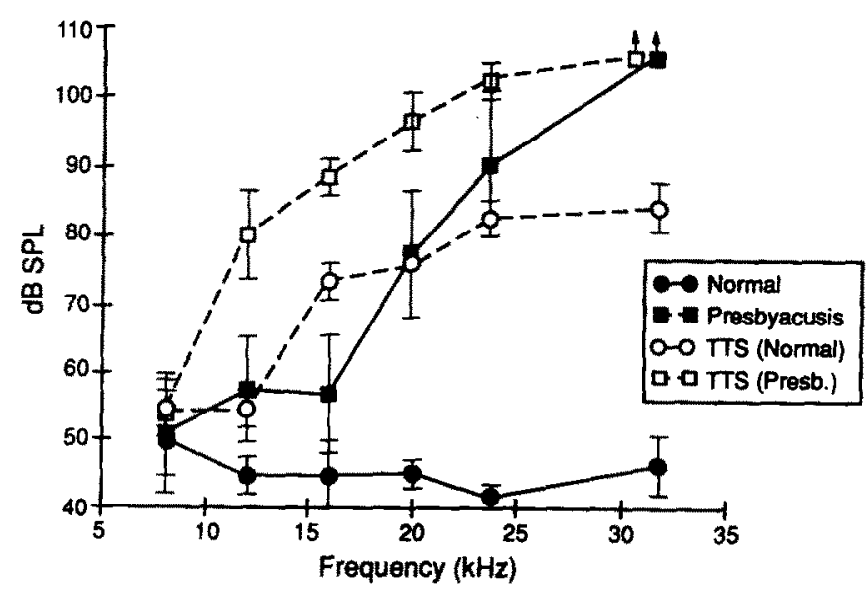

Fig. 4. Temporary threshold shift in six-month-old normal-hearing mice ( $\mathrm{CBA} / \mathrm{Ca})$ compared to mice with presbycusis $(\mathrm{C} 57 \mathrm{BL} / 6)$. Symbols with upward arrows represent the limit sound level of the system did not evoke a response. 


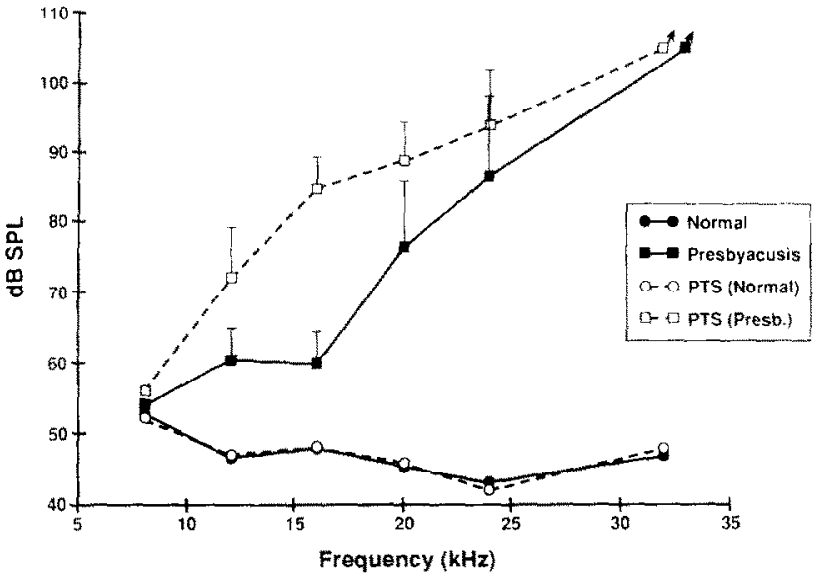

Fig. 5. Permanent threshold shift in six-month-old mice (CBA/Ca) compared to mice with presbycusis (C57BL/6). Upward arrows, see Fig. 4.

Fig. 5 shows pre-exposure sensitivity functions and those observed one week later for $\mathrm{CBA} / \mathrm{Ca}$ and C57BL $/ 6$ subjects. A significant PTS was observed for $\mathrm{C} 57 \mathrm{BL} / 6$ subjects for the grouped test frequencies above $8 \mathrm{kHz}$, excluding $32 \mathrm{kHz}$ at which frequency no threshold was measurable. Significant differences were observed for individual test frequencies at 16 and 24 $\mathrm{kHz}$. Thus, while this exposure produced no PTS in normal subjects, mice with premature presbycusis were susceptible to a noise-induced PTS (mean $=17 \mathrm{~dB}$, across $12-24 \mathrm{kHz}$ ).

\section{Hair Cell Loss}

Inner hair cell (IHC) loss was minimal in all subjects of all groups, and no significant IHC differences were

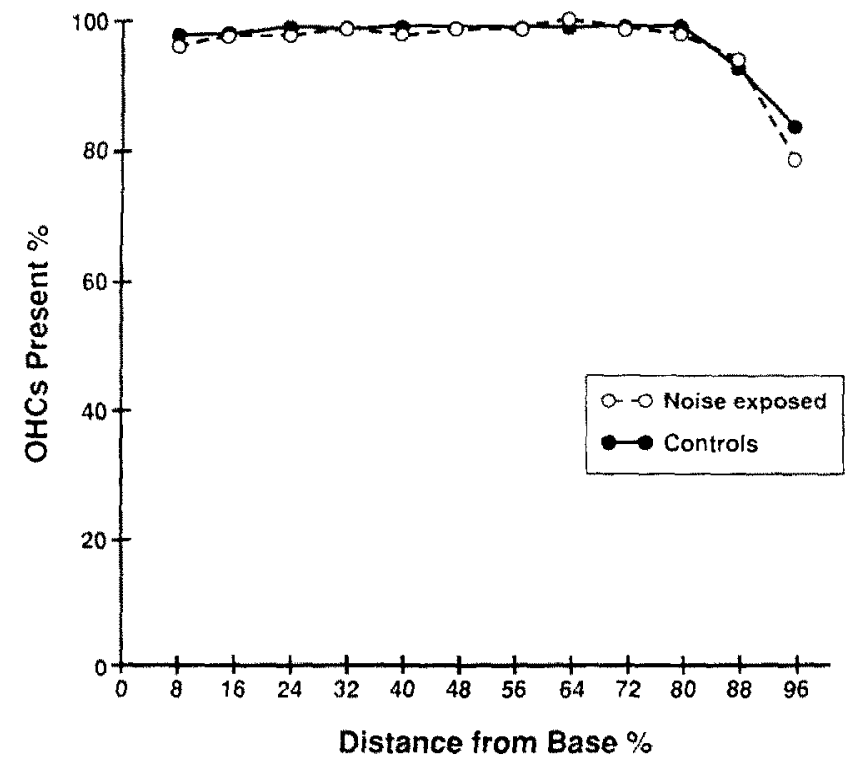

Fig. 6. The percent outer hair cell loss by location along the cochlear spiral (cytocochleogram) of a group of aged (21-month-old) $\mathrm{CBA} / \mathrm{Ca}$ mice receiving noise exposure (open circles) is compared to a group without noise exposure (closed circles)

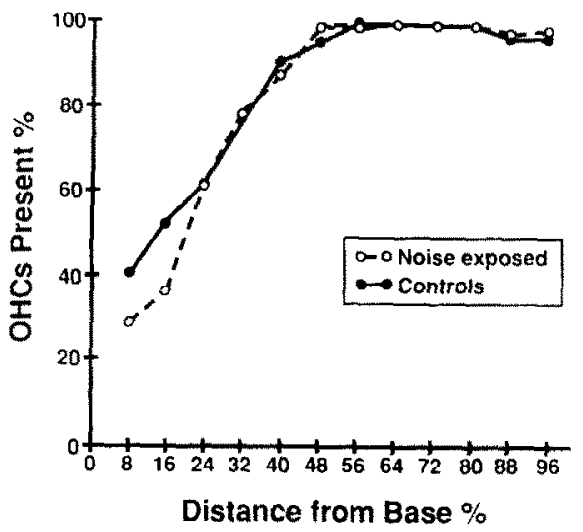

Fig. 7. The percent outer hair cell loss by location along the cochlear spiral (cytocochleogram) in a group of 6 -month-old CS7BL $/ 6$ mice after noise exposure (open circles) is compared with that is a group without noise exposure (closed circles).

observed. Cytocochleograms of six-month-old CBA/Ca mice showed completely intact inner and outer hair cell populations in both noise-exposed and control animals.

The cytocochleogram of the exposed and unexposed 21-month-old CBA/Ca mice (Fig. 6) showed occasional outer hair cell (OHC) losses throughout the cochlea, particularly marked at the apex. Thus, while these subjects showed baseline thresholds elevated by approximately $10 \mathrm{~dB}$ as compared to young CBA/Ca mice at higher frequencies, no corresponding basal $\mathrm{OHC}$ loss was observed in the cytocochleogram. Consistent with electrophysiological observations of no noise-induced PTS in the CBA/Ca animals of either age, there was no significant difference between the cytocochleogram of controls and that of noise-exposed CBA/Ca animals. Cytocochleograms of exposed and unexposed C57BL/6 mice (Fig. 7) showed OHC loss in the basal half-turn. This verified the ongoing presbycusis expected in these subjects. A somewhat reduced number of surviving OHC's were observed in the basal turn of noise exposed cochlea; however, this difference in the cytocochleograms between exposed and unexposed subjects did not reach statistical significance. It is possible that further OHC loss might have occurred with longer survival periods, however, there is good correlation between cytocochleograms and ABRs.

\section{Discussion}

The physiological results of this study demonstrate that mice having an age-related hearing loss, specifically $\mathrm{C} 57 \mathrm{BL} / 6$, are more susceptible to permanent noise-induced injury than similar aged subjects with normal-hearing, $\mathrm{CBA} / \mathrm{Ca}$. Moreover, in the absence of presbycusis, the aged cochlea does not demonstrate altered susceptibility to the noise levels used in this 
study. Our finding of normal hearing in CBA/Ca mice 600 or more days old are consistent with the literature (McGinn et al., submitted). Our measure of threshold yielded functions consistent with these recent findings, but elevated in overall level by $20 \mathrm{~dB}$. This appears to reflect calibration and earphone coupling procedures. It should not influence the measured TTS induced by noise exposure.

The anatomical results show small amounts of structural change with age, and no significant difference between the exposed and control groups. These findings are consistent with the view that hair cells can be affected by noise, so as to cause lack of function without sufficient structural damage to cause cell death. Hair cell counts are a relatively insensitive measure of noise-induced damage of functional significance, particularly that which results from low to moderate noise exposures. The noise exposure used in this study, 101 $\mathrm{dB}$ SPL for $45 \mathrm{~min}$, was sufficient to cause functional impairment, but, it was insufficient to cause degeneration visible by light microscopy. This level of exposure had been chosen because preliminary studies showed that the TTS produced was measurable at most test frequencies shortly after exposure. More severe noise exposure resulted in TTS changes of such magnitude that ABR responses could not be evoked at most frequencies, hence, it would not have been possible to compare the TTS in the different groups in a quantitative way. However, to assess the anatomical basis for the observed physiological changes, future anatomical studies will require use of higher noise levels than were used in this study, or more sensitive anatomical measures.

In Fig. 4 the TTS is represented by the area between the open squares versus closed squares (for presbycusis subjects), and the area between open and closed circles (for normal subjects). The smaller TTS observed in subjects with presbycusis, [having a pre-exposure hearing loss of $>28 \mathrm{~dB}$ (Fig. 4)] can be attributed to a number of factors. These include the biophysics of the exposure, the limitations of the auditory system, and the specific functional tests used in this study. Thus, for the high levels of stimulation required at 20 and $24 \mathrm{kHz}$ for the $\mathrm{C} 57 \mathrm{BL} / 6$ subjects ( $>95 \mathrm{~dB}$ ) to elicit a visually detectable ABR, the spectral spread of energy along the basilar membrane may have caused the sampling of activity from hair cells more toward apical regions of the cochlea. Since the apical areas were relatively unaffected by the noise exposure, spectral spread of the travelling wave would lead to a reduced estimate of the TTS.

More significant than the TTS differences are the PTS results of this study. In Fig. 5, one can see that the C57BL/6 presbycusis model develops significant permanent sensitivity change following noise exposure, whereas the normal subjects were unaffected. There are many reasons that may account for this phenomenon (i.e., a combinational effect of two or more factors such as age and noise) but caution must bc exercised in drawing conclusions from genetic models. Given the limits of our observations and the gross nature of the measure employed, it is pre-mature to characterize the nature of the interaction (e.g., additive). Nonetheless, the combinational response is one which may join those reviewed by Humes (1981) (i.e., noise combined with ototoxicity) and when pursued by further careful experiments will provide insight into pathophysiology.

The increased susceptibility of the ear with presbycusis to a noise-induced permanent threshold change is consistent with the clinical notion of a 'decreased cochlear reserve' in the older ear, that is a decreased metabolic responsiveness to recover from environmental and system stress. The current data of this study indicate that a 'decreased cochlear reserve' is not simply a function of age, but is dependent upon factors associated with obvious and measurable pathology. This view is also consistent with the expectation that other inner ear stresses, such as ototoxic agents will summate with noise to produce excess damage. The interaction of noise and ototoxicity has been studied by many investigators, as reviewed by Humes (1984). Although the results are highly dependent on dosages and time intervals, most results indicate that the combined effect is more damaging than either the noise or the ototoxicity agent alone.

\section{Conclusions}

Mice with an age-related hearing loss, perhaps analogous to human presbycusis, were more susceptible to permanent noise-induced hearing loss than normal mice. Aged mice without any sign of hearing loss had equivalent threshold shifts to those of normal mice. The results of these studies indicate that, subjects with presbycusis should take extra precautions to protect their ears against further damage from noise.

\section{Acknowledgements}

This grant was supported in part by NIH grant No. 1R01AG08885 and the TWJ Fellowship Program. We would also like to thank Denise D. Dyson for her assistance in the preparation of this manuscript.

\section{References}

Bennett, C.L., Davis, R.T. and Miller, J.M. (1983) Demonstration of presbycusis across repeated measures in a non-human primate species. Behav. Neurosci. 97, 602-607. 
Chole, R.A. and Henry, K.R. (1983) Disparity in the cylocochleogram and the electrocochleogram in aging $\mathrm{LP} / \mathrm{J}$ and $\mathrm{A} / \mathrm{J}$ inbred mice. Audiology 22, 384-392.

Church, M.W. and Shucard, D.W. (1986) Age related hearing loss in BDF1 mice as evidenced by the brain stem auditory evoked potential. Audiology 25, 363-372.

Crowley, D.E, Swain, R.E., Schramm, V.L. and Swanson, S.N. (1972) Analysis of age-related changes in electrical responses from the inner ear of rats. Ann. O.R.L. 81, 739-746.

Dum, N., Schmidt, U and von Wedel H. (1980a) Age-related changes in the auditory evoked brain stem potentials of albino and pigmented guinea pigs. Arch. Otorhinolaryngol. 228, 249-258.

Dum, N., Schmidt, U and von Wedel H. (1980b) Age-dependence of the neural auditory thresholds of albino and pigmented guinea pigs. Arch. Otorhinolaryngol. 229, 191-199.

Hawkins, J.E., Miller, J.M. and Rouse, R.C. (1985) Inner ear histopathology in aging rhesus monkeys. In: R.T. Davis (Ed.), Behavior and Pathology of Aging in Rhesus Monkeys, Alan Liss Publishers, New York, NY, pp. 137-154.

Henry, K.R. (1982a) Age related changes in the sensitivity of the post-pubertal ear to acoustic trauma. Hear. Res. 8, 285-294.

Henry, K.R. (1982b) Influence of genotype and age on noise-induced auditory losses. Behav. Genet. 12, 563-573.

Henry, K.R. (1982c) Age related auditory loss and genetics: an electrocochleographic comparison of six inbred strains of mice. J Gerontol. 37, 275-282.

Henry, K.R. (1983a) Lifelong susceptibility to acoustic trauma: changing patterns of cochlear damage over the lifespan of the mouse. Audiology 22, 372-383.

Henry, K.R. (1983b) Aging and audition. In: J.F. Willott (Ed.), The Psychobiology of the mouse, Charles C. Thomas, Springfield, IL. pp. $470-478$.

Henry, K.R. and Chole, R.A. (1980) Genotypic differences in behavioral, physiological and anatomical expressions of age-related hearing loss in the laboratory mouse. Audiology 19, 369-383.

Henry, K.R. and Lepkowski C.M. (1978) Evoked potential correlates of genetic progressive hearing loss. Age-related changes from the ear to the inferior colliculus of CS7BL $/ 6$ and CBA/J mice. Acta Otolaryngol. 86, 366-374.

Humes, L.E. (1984) Noise-induced hearing loss as influenced by other agents and by some physical characteristics of the individual. J. Acoust. Soc. Am. 76, 1318-1329.

Hunter, K.P. and Willott, J.F. (1987) Aging and the auditory brain stem response in mice with severe or minimal presbycusis. Hear. Res. 30, 207-218

Lutovats, M. (1969) Age in the occurrence and evaluation of acoustic trauma. Srpski. Archiv. Celokupno. Lekarstvo. 97, 869-873.

McGinn, M.D., Bean-Knudsen, D. and Ermel, R.W. (1991) Incidence of chronic otitis media in $\mathrm{CBA} / \mathrm{J}$ and $\mathrm{CBA} / \mathrm{CaJ}$ mice. Hear. Res.

McRae, J.H. (1971) Noise-induced hearing loss and presbycusis. Audiology 10, 323-333.

Mikaelian, D.O. (1979) Development and degeneration of hearing with C57/b16 mousc: relation of electrophysiologic responses from the round window and cochlear nucleus to cochlear anatomy and behavioral responses. Laryngoscope $89,1-15$.

Mills, J.H., Schmiedt, R.A. and Kulish, L.F. (1990) Age-related changes in auditory potentials of Mongolian gerbil. Hear. Res. $46,201-10$

Novotny, Z. (1975a) Age factor in auditory fatigue in occupational hearing disorders due to noise. Cesk. Otolaryngol. 24, 5-9.

Novotny, Z. (1975b) Development of occupational hearing loss after entering a noisy employment in older age. Cesk. Otolaryngol. 24, $151-154$.

Schmiedt, R.A., Mills, J.H. and Adams, J.C. (1990) Tuning and suppression in auditory nerve fibers of aged gerbils raised in quiet or noise. Hear. Res. 45, 221-36.

Shnerson, A. and Pujol, R. (1981) Age related changes in the C57BL $/ 6 \mathrm{~J}$ mouse cochlea. 1. Physiological findings. Dev. Brain Res. 2, 65-75.

Welleschik, B. and Raber, A. (1978) Einfluss von Expositionszeit und Alter auf den Iarmbedingten Horverlust. Laryngol. Rhinol. Otol. 57, 1037-1048.

Wenngren, B.I. and Anniko, M. (1988) A frequency specific auditory brain stem response technique exemplified in the determination of age related auditory thresholds. Acta. Otolaryngol. 106, 238243.

Willott, J,F. (1986) Effects of aging, hearing loss, and anatomical location on thresholds of inferior colliculus neurons in $\mathrm{C} 57 \mathrm{BL} / 6$ and CBA mice. J. Neurophysiol. 56, 391-408. 